\title{
GLOBAL SCALE ELECTRON PRECIPITATION DURING SUBSTORM EXPANSIONS
}

\author{
N. Østgaard, J. Stadsnes, J. Bjordal, E. Thorsen \\ Department of Physics, University of Bergen \\ N-5007 Bergen, Norway \\ R. R. Vondrak and S. A. Cummer \\ Laboratory for Extraterrestrial Physics, Goddard Space Flight Center, \\ Greenbelt, MD 20771 \\ D. L. Chenette \\ Lockheed-Martin Advanced Technology Center \\ Palo Alto, CA 94304 \\ G. K. Parks and M. J. Brittnacher \\ Geophysics Program, University of Washington \\ Seattle, WA 98195 \\ D. L. McKenzie \\ The Aerospace Corporation \\ Los Angeles, CA 90009-2957
}

\begin{abstract}
The Polar Ionospheric X-ray Imaging Experiment (PIXIE) and the Ultraviolet Imager (UVI) on the POLAR satellite have provided the first simultaneous global scale views of the patterns of electron precipitation through imaging of the atmospheric X-ray bremsstrahlung and the auroral UV emissions. While UVI responds to the total electron energy input, PIXIE responds only to the high energy (multi-keV) electron precipitation. During the substorm expansion phase, clear time delays occur between the electron injection at the nightside and the start of the precipitation on the morning/dayside. The observations are generally consistent with patterns previously deduced from ground-based and suborbital observations.
\end{abstract}

\section{Introduction}

Previous studies by ground based, balloon and space observations have indicated that the patterns of energetic electron precipitation differ significantly from those found in the visible and the UV auroral oval [Akasofu, 1968]. But due to the lack of global imaging of the energetic electron precipitation, one has not been able to establish a complete picture. This study focuses on the development of the electron precipitation during the different phases of magnetospheric substorms. The prominent features of the high energy precipitation seen in the $\mathrm{X}$-ray images are compared to those of the lower energy precipitation seen in the UV images.

Based on the images from PIXIE [Imhof et al., 1995] and UVI [Torr et al., 1995] on board the POLAR satellite we have studied isolated and multiple substorms. The POLAR satellite was launched 24 February 1996 into a 1.8 x $9 \mathrm{R}_{\mathrm{E}}$ polar orbit. During the apogee passes the imagers operate for about 12 hours. To avoid damage, the imagers must be turned off while the satellite passes through the radiation belt. Restricted by this operating time we have examined the data from 1996 to select events where the imagers were operating during the entire substorm event. Fifteen events from 9 days complied with this requirement. Only data from the PIXIE rear chamber, which measures X-rays from 10 to $20 \mathrm{keV}$, were used for this study. These X-rays are produced by energetic electrons with minimum energies of 10 to $20 \mathrm{keV}$. UV emissions in the LBH long band are proportional to the total energy flux of the precipitating electrons [Germany et al., 1994] with energies down to the excitation energy $(7 \mathrm{eV})$. Usually electrons of energy less than $10 \mathrm{keV}$ dominate in this energy flux.

In this paper we show observations of the substorm of 12 September 1996, an event generally representative of many features commonly found in the full set of data. 


\section{Substorm Observations on 12 September 1996}

The substorm observations on September 12, 1996 by PIXIE and UVI are shown in Figure 1. The X-ray images are summed over 10 minutes, as indicated in each panel. The UVI image in the LBHL band for the central time of each interval is shown in the right hand panel. The X-ray and UV images are displayed on a linear scale, with a maximum UV brightness (denoted by red) corresponding to 50 photons $\bullet \mathrm{cm}^{-2} \bullet \mathrm{s}^{-1}$, and a maximum X-ray flux (denoted by dark red) corresponding to 350 photons $\bullet\left(\mathrm{keV} \bullet \mathrm{s} \bullet \mathrm{sr}^{\bullet} \mathrm{cm}^{2}\right)^{-1}$ in the 10 to $20 \mathrm{keV}$ energy range. The counting statistics yield an uncertainty in the X-ray flux of approximately $15 \%$.

The X-ray images contain grids of geomagnetic latitude and longitude, and the midnight-noon and dawn-dusk MLT meridians are marked by solid lines. Magnetic local times are marked in red text. The day-night terminator at ground level is marked by the bold dashed line. In the two first Xray images, the position of the geosynchronous LANL 1994 satellite mapped into the ionosphere at $100 \mathrm{~km}$ height is shown as a red circle (traced along the field using Tsyganenko 1989 model using WIND observations for the appropriate inputs). The UV images contain grids of geographic latitude and longitude, but the two sets of images are aligned with respect to each other, allowing a direct comparison of the positions of the auroral emissions.

The substorm onset occurred at about 13:46 UT, as indicated in the UVI image. This is only dimly visible in the 13:40-13:50 UT X-ray image. However, by accumulating for 5 minutes all $\mathrm{X}$-rays originating in a small box around the known onset region and stepping 30 seconds for next count, we can find the precipitation onset from the X-ray images. The X-ray intensity accumulated in this manner as a function of time is shown in Figure 2, and there is a clear precipitation onset between 13:45 and 13:46 UT, corresponding well to the observed UVI onset time. This timing also agrees well with geosynchronous energetic particle data from the LANL 1994 satellite, which is also shown in Figure 2. This satellite observed an injection of protons at 13:46 UT, when the satellite is located at 20.8 MLT, just southwest of the injection region.

From 14:00-14:40 UT, a poleward bulge is seen in both $\mathrm{X}$-rays and UV. However, the $\mathrm{X}$-rays are most intense at the poleward edge of the UV emission region, indicating more energetic precipitation at the poleward edge. From 13:50-14:20 UT, the X-ray images also show a slight westward motion of the precipitation region. This feature is common in the $\mathrm{X}$-ray images and is often more dramatic than in this event.

A notable feature in the X-ray images is energetic particle precipitation from approximately 0400 to 1100 UT delayed relative to the onset of the substorm. This is likely due to the eastward drift and subsequent precipitation of the electrons injected at substorm onset. We have accumulated the X-ray counts in a region centered at 06 MLT, and this variation of X-ray intensity with time is shown in Figure 2. There is a clear precipitation onset between 14:05 and 14:06 UT, which gives a delay from onset of 19 minutes for drift from 21 MLT to 6 MLT, in turn giving $57 \mathrm{~min}$. for one drift period assuming constant drift velocity. Using $\mathrm{L}=6.6 \mathrm{Re}$ (geosynchronous orbit) as the onset region, a rough estimate of the energy of the drifting electrons responsible for the observed morning precipitation is $100 \mathrm{keV}$, which is more than sufficient to produce $\mathrm{X}$-rays in the energy range observed by PIXIE.

As can be seen in the X-ray images from 14:20 to 15:00 UT, this enhanced morning sector precipitation is not smoothly distributed in local time but rather is strongly concentrated around 07 MLT. Although this region is at the very edge of the field of view of the UVI instrument, this feature appears to be absent from the UV images, further supporting the conclusion that high energy electrons are creating the observed X-ray emissions. Possible causes of this localized precipitation are discussed briefly below.

\section{Discussion and Conclusions}

In this paper we report the first results of a study of the global features of the energetic electron precipitation during substorms. As shown in the data from 12 September 1996, three common features are seen:

- A poleward bulge of energetic electron precipitation during the break-up. The brightest X-ray emissions are at the poleward edge of the UV emissions, indicating higher energy precipitation at this poleward edge.

- A westward motion of the precipitation region occurring after precipitation onset.

- Intensified precipitation at 04-11 MLT delayed relative to the night sector onset time. The delay time corresponds to the magnetic field gradient drifting time of approximately $100 \mathrm{keV}$ electrons. This energy is consistent with the emission of X-rays in the observed $10-20 \mathrm{keV}$ energy range.

We have examined 15 events from 1996 and found these common features in 12 of them. In 3 events we did not see any intensified precipitation into the morning sector, most probably because the events were too weak. These observations of enhanced morning precipitation indicate the existence of a mechanism in the morning sector that provides pitch angle diffusion of the energetic electrons 

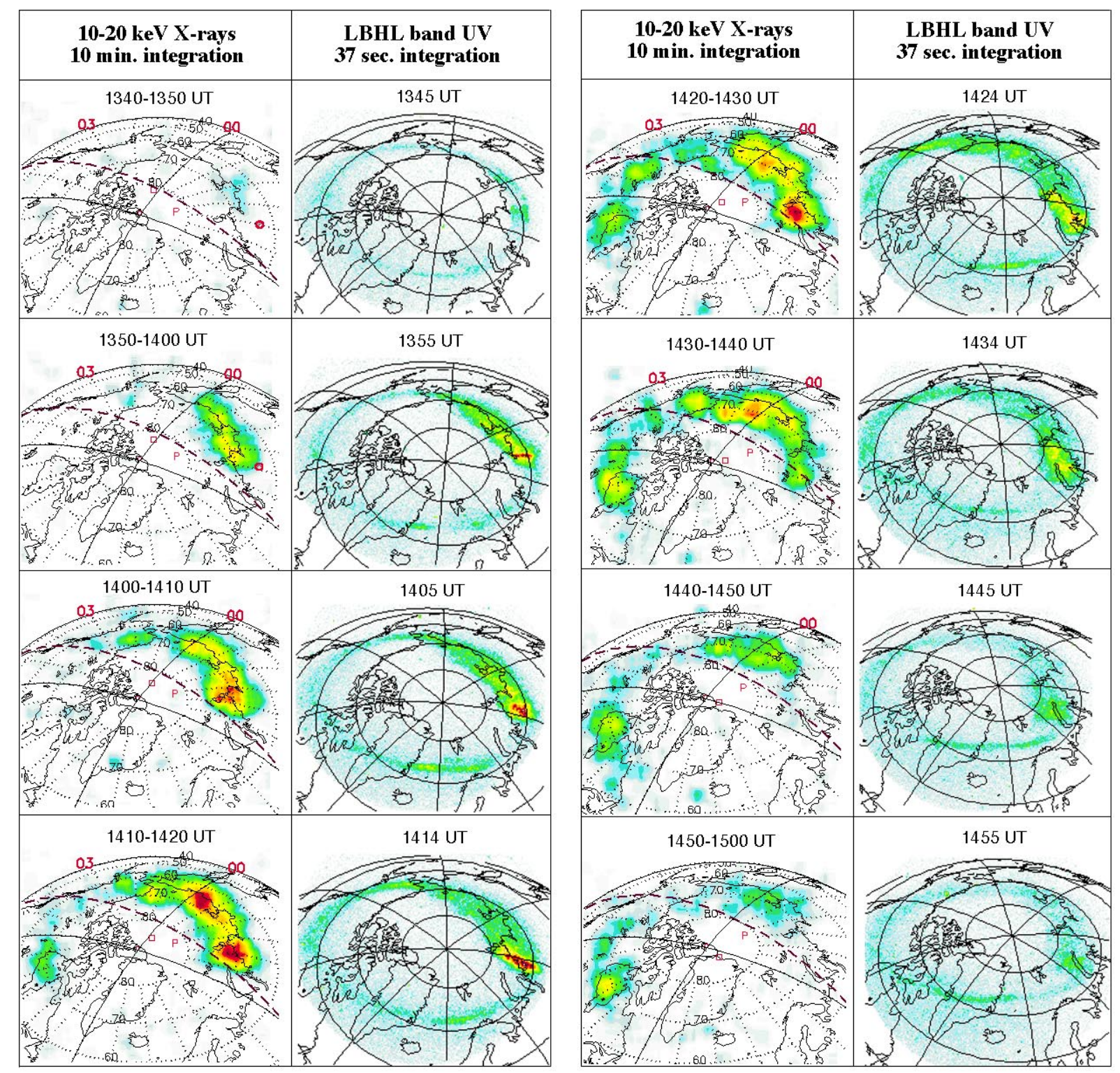

Figure 1. Simultaneous X-ray and UV images showing the development of the 12 September 96 substorm.

into the loss cone. One candidate for such a mechanism is the wave- particle interaction of VLF waves and electrons, which has the cold plasma density as an important parameter. As we see, the precipitation often increases just after the electrons enter the sunlit part of their orbit, this feature will be investigated more thoroughly in the further study.

While PIXIE provides the first simultaneous and global view of the X-ray aurora, previous studies have assimilated local observations to form a statistical global picture [Akasofu, 1968; Berkey et al., 1974; Miller and Vondrak, 1985]. Figure 3 is a reproduction of a figure from Akasofu
[1968] which shows the development of the X-ray substorm as inferred from ground-based balloon measurements. The general morphology is remarkably similar to the PIXIE observations, although the PIXIE images show some new features (e.g. the enhanced morning precipitation region) and allow a direct event-based comparison with observations from other instruments.

Acknowledgments. The work at University of Bergen was supported by the Norwegian Research Council (NFR). The work at Lockheed Martin was supported under NASA contract NAS-5-30372. The work at the Aerospace 

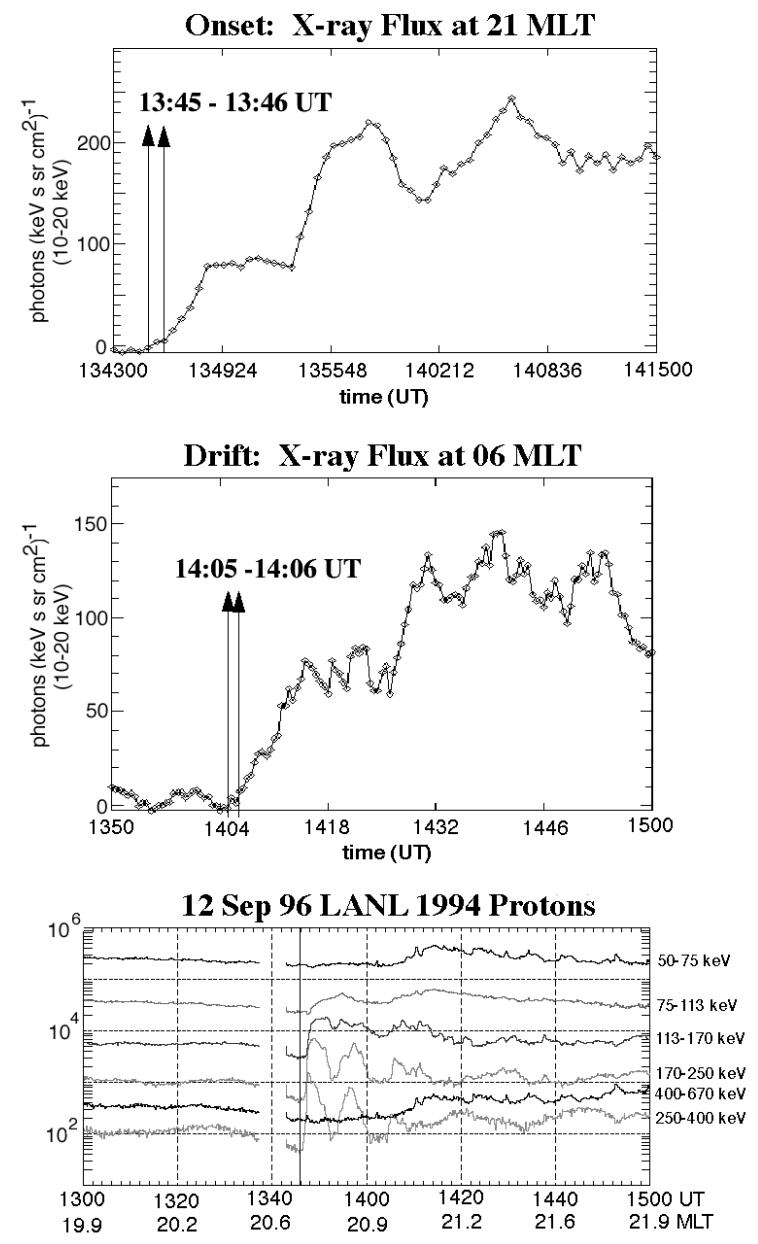

Figure 2. The temporal development of the 21 MLT sector precipitation, the 06 MLT sector precipitation, and the injected protons observed at geosynchronous orbit.

Corporation was supported under NASA contract NAS-530369. The authors thank Dr. Geoff Reeves at Los Alamos National Laboratory for providing data from the geosynchronous satellites.

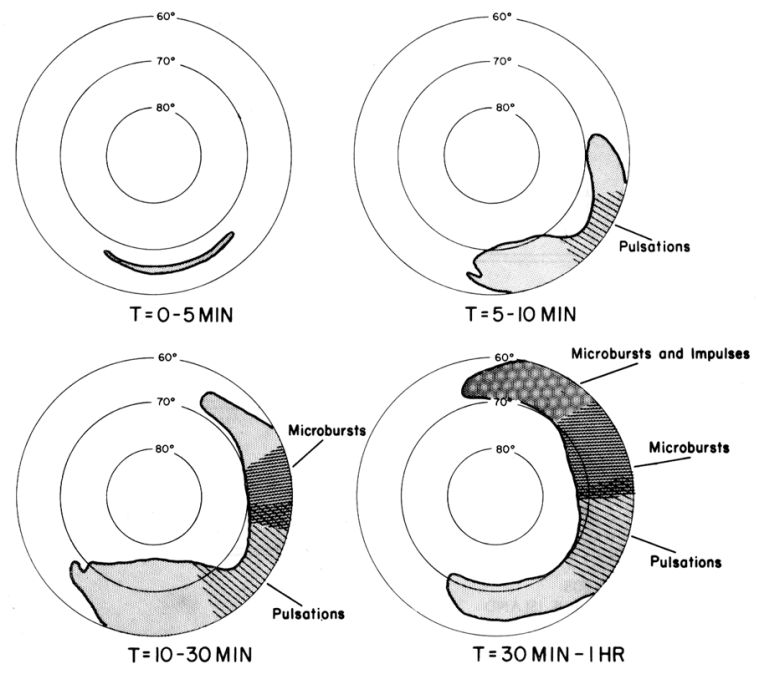

Figure 3. The development of the X-ray substorm inferred from ground-based instruments [Akasofu, 1968].

\section{References}

Akasofu, S.-I., Polar and Magnetospheric Substorms, New York: Springer-Verlag, 1968

Berkey, F. T. et al., A synoptic investigation of particle precipitation dynamics for 60 substorms in IQSY (1964-65) and IASY (1969), Planet. Space Sci., 22, 255, 1974.

Germany, G. A. et al., The use of FUV auroral emissions as diagnostic indicators, J. Geophys. Res., 99, 383, 1994.

Imhof, W. A. et al., The Polar ionospheric X-ray imaging experiment (PIXIE), Space Sci. Rev., 71, 385, 1995.

Miller, K. L., and R. R. Vondrak, A high-latitude phenomenological model of auroral precipitation and ionospheric effects, Radio Sci., 20, 341, 1985.

Torr, M. R. et al., A far ultraviolet imager for the international solar-terrestrial physics mission, Space Sci. Rev., 71, 329, 1995.

Correspondence to: N. Østgaard, Department of Physics, University of Bergen, Allegaten 55, N-5007 Bergen, Norway, e-mail: nikolai.ostgaard@fi.uib.no) 